\title{
Perception and Outcome of PEM Fellows following POCUS Curriculum Implementation
}

\author{
Steven Dawson and Ben Nti \\ Indiana University School of Medicine
}

Introduction: Point-of-care ultrasonography (POCUS) in the emergency department provides the clinician with a real-time, non-invasive and rapid assessment of their patients. Currently, there is a lack of published POCUS curriculum guidelines for pediatric emergency medicine (PEM) trainees. In this regard, we developed a POCUS curriculum for fellows at a newly established PEM fellowship at our institution to understand their perception and its effectiveness.

Methods: We enrolled all current PEM fellows $(n=4)$ who had limited to no prior experience with POCUS to participate in program implementation in January 2018. Their progress was followed over time until graduation. The curriculum included didactics hands-on training in the presence (supervised) and absence (solo) of an expert instructor. In addition to the curriculum, the fellows were also required to complete a pre- and post-survey assessment.

Results: Before curriculum implementation, barriers of POCUS common to all fellows (100\%) included image interpretation and comfort level. Conversely, this improved with only 1 fellow (25\%) suggesting these barriers. Interestingly, nearly all fellows $(75 \%)$ found POCUS to be time-consuming following the curriculum implementation. Knowledge-base assessment showed improvement from an average score of $50 \%$ pre-test versus $79 \%$ post-test. The number of scans increased with time, but qualitative measures of image acquisition had a minimal improvement on a self-assessment quality assurance (QA) Likert scale. Average solo QA rating by an expert was higher (Mean 3.23 + SEM 0.15) than the average by the fellows' selfassessment $(3.07+0.18)$. Overall sensitivity of POCUS examinations was $90.32 \%(61.92 \%$ $93.07 \%)$ and specificity was $99.11 \%$ (94.07\%-99.57\%).

Conclusion: POCUS curriculum implementation can improve the PEM fellow's skills while enhancing their experience. While there are challenges for the utilization of POCUS, this study shows that the implementation of such a curriculum may have a positive effect on skill, knowledge development as well as perception. 